\section{R.TH Revista \\ Argentina de Antropología Biológica}

TRABAJO ORIGINAL

\section{Condiciones de salud de una población antigua del occidente de Mesoamérica: Estudio de los habitantes del sitio arqueológico Los Limones, México (600- 1000 d.C.)}

\section{Health condition of an ancient population in the west of Mesoamerica: study of the inhabitants of Los Limones archaeological site, Mexico (600-1000 d.C.)}

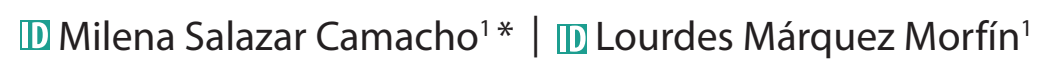

1) Posgrado en Antropología Física, Escuela Nacional de Antropología e Historia.

REVISTA ARGENTINA DE ANTROPOLOGÍA BIOLÓGICA

Volumen 24, Número 1

Enero-Junio 2022

Financiamiento: Beca de excelencia de la Secretaría de Relaciones Exteriores, Gobierno de México.

*Correspondencia a: Milena M. Salazar Camacho. Posgrado en Antropología Física, Escuela Nacional de Antropología e Historia. Periférico Sur y Calle Zapote S/N, Isidro Fabela, Tlalpan, 14030 Ciudad de México, México.

Email: mmsc13@hotmail.es

RECIBIDO: 10 NOVIEMBRE 2020

ACEPTADO: 9 JUNIO 2021

https://doi.org/10.24215/18536387e043 e-ISSN 1853-6387

https://revistas.unlp.edu.ar/raab

\section{Resumen}

Esta investigación evalúa las condiciones de salud de 29 individuos que habitaron el sitio Los Limones durante la fase Armería (600-1000 d.C.) para determinar si tenían alguna relación con la subsistencia agrícola que se ha propuesto para este sitio. Con base en la metodología estandarizada de indicadores de estrés, se realizó un análisis macroscópico de cuatro lesiones óseas y seis lesiones dentales. Las caries, los cálculos dentales, la pérdida de piezas dentales ante mortem y las reacciones periostales fueron las lesiones más frecuentes y se identificaron en individuos juveniles, adultos jóvenes y adultos de ambos sexos. La integración de los datos del medio ambiente, del contexto arqueológico y de la identificación de las lesiones óseas y dentales permitió sugerir que los antiguos habitantes del sitio Los Limones pudieron haber tenido una estrategia de subsistencia mixta que integró actividades como la agricultura, la pesca, la caza y la recolección de plantas y frutos silvestres. https://doi.org/10.24215/18536387e043

Palabras clave: salud, agricultura, estrategia de subsistencia mixta, sitio Los Limones, México.

\section{Abstract}

This research focuses on the health condition of 29 individuals that inhabited Los 
Limones site during the Armería phase (600-1000 AD). The individuals were evaluated to determine if their health conditions had any relationship with the agricultural subsistence practices that have been proposed for the site. On the basis of the standardized methodology for calculating stress indicators, a macroscopic analysis of four bone and six dental lesions was conducted. Dental caries, calculus, antemortem tooth loss, and periosteal reactions were the most frequent lesions. These lesions were identified in juvenile, young adult, and adult individuals of both sexes. Data derived from the environmental and archaeological context, as well as from the identification of bone and dental lesions, suggest that the ancient inhabitants of the Los Limones site may have had a mixed subsistence strategy that included activities such as agriculture, fishing, hunting, and collecting wild plants and fruits. https://doi.org/10.24215/18536387e043

Keywords: health, agriculture, mixed subsistence strategy, Los Limones site, Mexico

Algunas condiciones y estilos de vida pueden ocasionar alteraciones en la salud y en el bienestar de una población. Por ejemplo, el surgimiento y la intensificación de la agricultura tuvo un impacto en la variabilidad biológica y cultural de las poblaciones antiguas de diferentes regiones del mundo. De manera general, las investigaciones bioarqueológicas han reportado que las poblaciones que adoptaron la agricultura como principal modo de subsistencia presentan un deterioro en la salud, el cual se ha reflejado en el incremento de lesiones óseas y dentales, la modificación de los patrones de crecimiento y estatura, la disminución de la calidad nutricional de la dieta, las variaciones en los perfiles demográficos, las modificaciones en los patrones de robustez, entre otros aspectos (Cohen y Armelagos, 1984; Cohen y Crane-Kramer, 2007; Larsen, 1995, 2006; Pinhasi y Stock, 2011). Estas investigaciones han brindado aportes importantes, pero es pertinente considerar que realizar inferencias sobre la salud de las poblaciones antiguas representa un desafío (Wood et al., 1992).

El sitio Los Limones ha sido considerado como una aldea agrícola del Occidente de Mesoamérica, ya que se localiza en las tierras más fértiles del valle de Colima (Olay y López, 2008). A pesar de que el Occidente, especialmente la región Balsas-Jalisco, ha brindado datos relevantes para comprender el origen de la agricultura y la domesticación de plantas en América (Piperno et al., 2009; Ranere et al., 2009; Zizumbo-Villareal y ColungaGarcía Marín, 2010; Zizumbo-Villarreal et al., 2012), para el valle y el estado de Colima son pocos los estudios publicados que hacen referencia a la subsistencia agrícola. Entre estos se pueden mencionar el hallazgo de una mazorca carbonizada en el sitio El Tropel (Jácome y Morland, 2011), los análisis de isótopos que evidenciaron el consumo de maíz en el sitio El Pantano (Beekman, 2010), y la cerámica utilitaria de la fase Capacha (1500500 a.C.) que hace referencia al cultivo, almacenamiento, transformación y consumo de plantas domesticadas (Kelly, 1980). Por lo tanto, es necesario desarrollar investigaciones más detalladas acerca de las estrategias de subsistencia que tuvo este valle para generar propuestas más sólidas que demuestren que sus antiguos habitantes dependían principalmente de la agricultura.

En esta investigación se evaluaron las condiciones generales de salud de 29 individuos que habitaron el sitio Los Limones durante la fase Armería (600-1000 d.C.). Tomando en cuenta que la salud es un estado multifactorial, se utilizó el enfoque biocultural para considerar la interacción dinámica entre los seres humanos y sus amplios entornos 
sociales, culturales y físicos (Zuckerman y Armelagos, 2011). Para lograr este propósito, se seleccionaron cuatro lesiones óseas y seis lesiones dentales para comprobar si tenían alguna relación con la subsistencia agrícola propuesta para el sitio, o si se atribuyen a otras estrategias de subsistencia y/o factores. Los resultados obtenidos fueron comparados con otros sitios arqueológicos de la cuenca de México y el área Maya, ya que se ha publicado poca información bioarqueológica de los antiguos habitantes de Colima.

\section{Ubicación del sitio Los Limones y sus características ambientales}

El sitio Los Limones se ubica sobre el margen izquierdo del arroyo Los Limones en el valle de Colima, estado de Colima, México (Fig.1). El terreno que abarca este sitio forma parte de las tierras agrícolas más productivas del suroeste del valle, las cuales han sido nutridas gracias a los depósitos de cenizas volcánicas (Almendros y González, 2009).

Este valle está atravesado de norte a sur por el río Armería y sus afluentes. Además, cuenta con lagunas y algunos arroyos (Instituto Nacional para el Federalismo y Desarrollo Municipal 2016). Durante la época prehispánica, los arroyos estacionales de poca profundidad pudieron haber sido desviados hacia las áreas cultivadas (López, 2011; Olay y López, 2008).

El valle de Colima se encuentra a una altitud que varía entre los 1700 y los $400 \mathrm{msnm}$. Esto genera una diversidad de climas, flora y fauna y, por lo tanto, ofrece una gran variedad de recursos (Almendros y González, 2009). Estas características indican que esta región representó un lugar propicio para los asentamientos antiguos, hecho que se ha demostrado con el hallazgo constante de materiales culturales (López, 2011; Olay y López, 2008).

\section{Contexto arqueológico}

Occidente es el área cultural más grande de Mesoamérica, pero a pesar de su riqueza

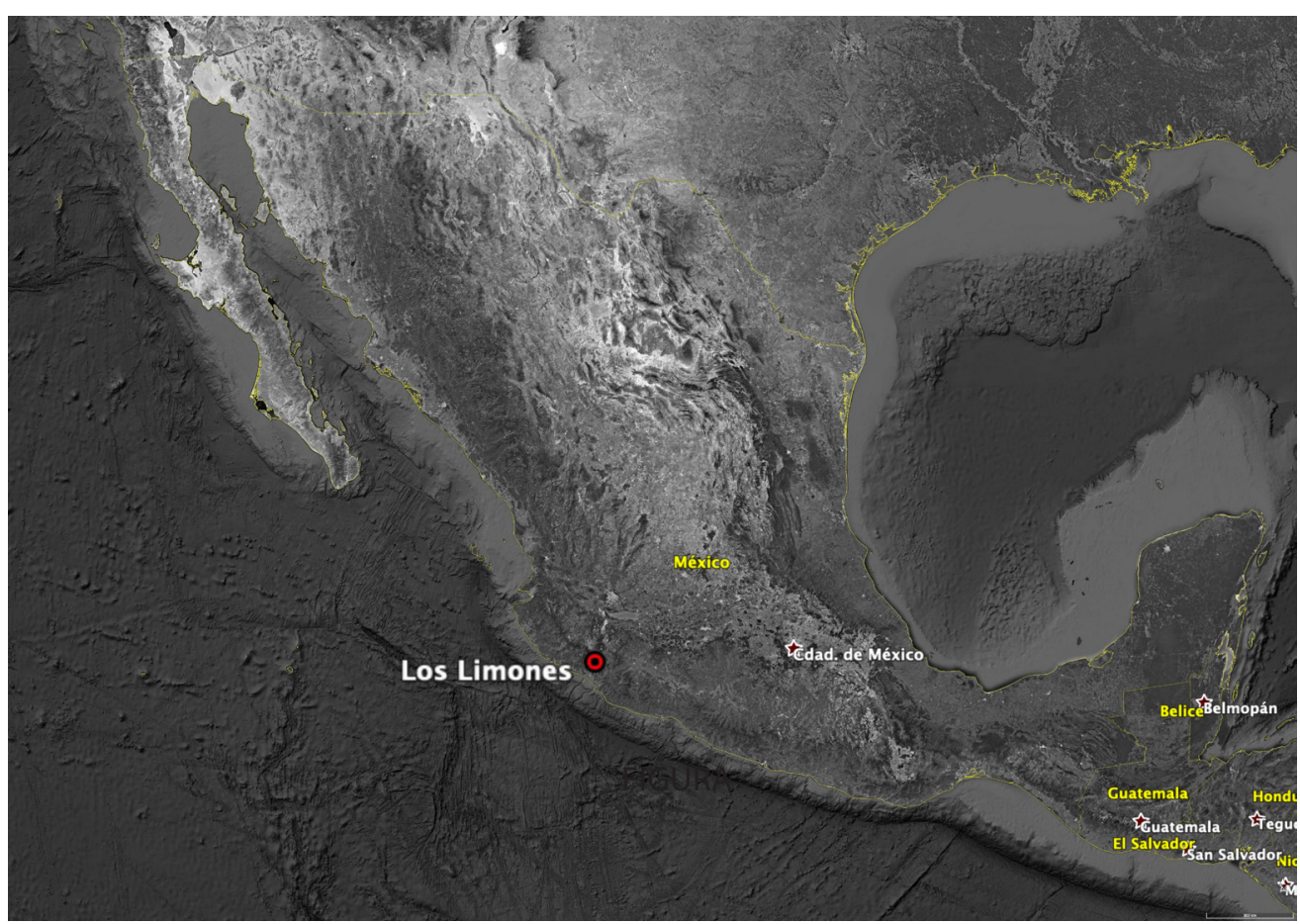

FIGURA 1. Ubicación del sitio arqueológico Los Limones. 
cultural y de su extensión geográfica, ha sido una de las regiones menos exploradas e investigadas (Almendros y González, 2009). En los últimos años, el aumento de la población y la consecuente expansión de las ciudades ha promovido el descubrimiento de sitios arqueológicos. Bajo este escenario, en el año 2007 se excavó el sitio Los Limones, como parte de un rescate arqueológico impulsado por un proyecto urbanístico.

El informe arqueológico ofrece información general de los principales hallazgos: una posible unidad habitacional y los contextos funerarios de la fase Armería (600-1000 d.C.). Además, enfatiza en el análisis de los materiales cerámicos y líticos recuperados (Olay y López, 2008), pero no brinda una interpretación detallada e integral del sitio ni de los individuos que lo habitaron.

\section{Unidad habitacional y zonas funerarias}

En la unidad de exploración 2 (U.E.2) se excavaron alineamientos de roca que pudieron ser los cimientos de una unidad habitacional. Los entierros 1 y 2 se descubrieron adyacentes a uno de esos alineamientos, ya que una de las costumbres funerarias de las fases Armería y Chanal (600-1500 d.C.) consistía en enterrar a los individuos dentro de las residencias. La particularidad de estos entierros radica en la importante cantidad de materiales cerámicos asociados como, por ejemplo, las figurillas de estilo Mazapa del Altiplano Central de México (Olay y López, 2008).

Los demás individuos analizados en esta investigación fueron recuperados en zonas funerarias (U.E. 3 y 7) con enterramientos característicos de la fase Armería (600-1000 d.C.) (López, 2011; Olay y López, 2008). La principal característica de estos enterramientos era que presentaban alineamientos y agrupamientos de rocas que reciben el nombre de "marcadores". Estos podían formar cuadros, lápidas o pisos que indican la ubicación de un enterramiento o de una ofrenda (López, 2011).

La mayoría de los artefactos asociados a estos enterramientos fueron vasijas cerámicas de tipo doméstico de la fase Armería (600-1000 d.C.). En menor cantidad se depositaron artefactos líticos como metates y manos de moler (Olay y López, 2008).

\section{MATERIALES Y MÉTODOS}

En esta investigación se analizaron macroscópicamente 29 individuos recuperados en las unidades de exploración 2, 3 y 7 (Olay y López, 2008). El estado de conservación de estos restos óseos es deficiente y varía de regular a malo, ya que los individuos se encuentran incompletos y fragmentados. Las piezas dentales tuvieron una mejor conservación, así como algunos fragmentos de huesos largos y del cráneo.

\section{Estimación de la edad a la muerte y determinación del sexo}

La edad de los individuos subadultos se estimó utilizando la secuencia de formación y desarrollo de los dientes (Ubelaker, 1978) y la fusión de las epífisis (Schaefer et al., 2009). En los individuos adultos, la edad se estimó con base en el desgaste dental (Lovejoy, 1985), los cambios en la sínfisis púbica (Brooks y Suchey, 1990) y las modificaciones en la superficie auricular (Buckberry y Chamberlain, 2002).

El sexo de los individuos adultos se determinó por medio del análisis macroscópico de las características morfológicas de la pelvis y el cráneo (Klales et al., 2012; Walker, 2005, 2008). En el caso de los individuos menores de 12 años, no se determinó el sexo debido a la falta de técnicas confiables (Scientific Working Group for Forensic Anthropology, 2010). 


\section{Identificación de lesiones óseas y dentales (indicadores de estrés)}

Una lesión es una anormalidad patológica asociada a una enfermedad o herida (Manchester et al., 2016). Las lesiones óseas y dentales han sido utilizadas para reconstruir patrones de salud (Goodman y Martin, 2002), ya que hacen referencia a patologías dentales, enfermedades metabólicas, deficiencias nutricionales, enfermedades infecciosas, alteraciones en el crecimiento y el desarrollo biológico, entre otros problemas de salud (Roberts, 2015).

En esta investigación se seleccionaron 10 lesiones y/o anormalidades: caries, cálculos dentales, pérdida de piezas dentales ante mortem, lesiones periapicales, periodontitis, hipoplasia del esmalte, reacciones periostales, criba orbitaria, exostosis auditiva y lesiones osteoarticulares. El registro y el análisis de estas lesiones y/o anormalidades se realizó con base en las metodologías estandarizadas (Buikstra y Ubelaker, 1994; Goodman y Martin, 2002; Roberts y Connell, 2004; Steckel et al., 2002, 2011), las cuales se ajustaron al estado de conservación de la colección osteológica.

Estas lesiones y/o anormalidades se analizaron de manera macroscópica con asistencia de una lupa de $2 X$ de aumento. Se contabilizó la frecuencia y el porcentaje de individuos que presentaron cada lesión y/o anormalidad, las cuales se registraron de la siguiente manera: (1) No observable: la pieza dental o el hueso necesario para evaluar la lesión no se conservó o las alteraciones tafonómicas no permitieron la evaluación; (2) Ausente: la pieza dental o el hueso se evaluó y la lesión no se presentó; (3) Presente: la pieza dental o el hueso se evaluó y la lesión estaba presente.

Las caries se evaluaron en los dientes permanentes y deciduos a partir de la presencia de una cavidad que afectara el esmalte dental (Hillson, 2001; Vega y Cucina, 2011). El cálculo dental también se identificó en piezas dentales permanentes y deciduas (Hillson, 2008; Roberts y Connell, 2004). La pérdida de piezas dentales ante mortem se registró con base en la presencia de reabsorción alveolar (Steckel et al., 2002; 2011; Vega y Cucina, 2011). Las lesiones periapicales se evaluaron en alvéolos de dientes permanentes y deciduos (Buikstra y Ubelaker 1994; Ogden, 2008; Steckel et al., 2002; 2011). La periodontitis se observó en los márgenes bucales de los alvéolos de los dientes permanentes posteriores (premolares y molares) (Ogden, 2008). La hipoplasia del esmalte se analizó en incisivos y caninos superiores e inferiores permanentes y sólo se registró el defecto lineal horizontal (Steckel et al., 2011).

En cuanto a las lesiones óseas, las reacciones periostales se evaluaron en huesos largos y se tomó en cuenta el tipo de hueso formado (reticular o lamelar) y el estado de la lesión (activa o inactiva) (Roberts y Connell, 2004; Weston, 2012). Se analizó la presencia de criba orbitaria en al menos una de las órbitas (Steckel et al., 2011) y se tomó en cuenta si la lesión estaba activa o inactiva (Klaus, 2017). La exostosis auditiva se registró en al menos uno de los canales auditivos (Steckel et al., 2002). Por último, solo fue posible identificar lesiones osteoarticulares en las vértebras y se registró la presencia de osteofitos en los cuerpos vertebrales (Roberts y Connell, 2004; Steckel et al., 2002).

\section{Análisis estadísticos}

Debido a que la muestra de estudio es pequeña, se utilizó la prueba exacta de Fisher para realizar comparaciones entre todas las lesiones y/o anormalidades óseas y dentales identificadas (caries, cálculos dentales, pérdida de piezas dentales ante mortem, lesiones periapicales, periodontitis, hipoplasia del esmalte, reacciones periostales, criba orbitaria, exostosis auditiva y lesiones osteoarticulares). Las comparaciones se realizaron entre: (1) femeninos-masculinos; (2) infante-juvenil; (3) infante-adulto joven; (4) infante-adulto; (5) 
juvenil-adulto joven; (6) juvenil-adulto y; (7) adulto-adulto joven. En todas estas pruebas se excluyó a los individuos de sexo indeterminado.

Los análisis se realizaron usando el paquete estadístico R (R Core Team, 2019) y la significancia estadística de todas las pruebas se determinó a partir del valor de $p \leq 0,05$.

\section{RESULTADOS}

\section{Estimación de la edad a la muerte y determinación del sexo}

Se utilizaron cuatro categorías de edad: (1) infante (nacimiento -1año); (2) juvenil (219 años); (3) adulto joven (20-30 años) y; (4) adulto (>30 años). De tal manera, se identificaron un infante $(3,4 \%)$, tres juveniles $(10,3 \%)$, cinco adultos jóvenes $(17,2 \%)$ y 20 adultos $(69,0 \%)$ (Tabla 1$)$.

Con respecto a la determinación del sexo, se establecieron tres categorías: (1) femenino, (2) masculino y (3) indeterminado. Del total de individuos analizados, se identificaron 10 femeninos (34,5\%), cuatro masculinos $(13,8 \%)$ y $15(51,7 \%)$ individuos de sexo indeterminado (Tabla 1).

Tabla 1. Distribución de los individuos según el sexo y la categoría de edad ( $n=29)$

\begin{tabular}{ccccccccc}
\hline Categoría de edad & \multicolumn{2}{c}{ Femeninos } & \multicolumn{2}{c}{ Masculinos } & \multicolumn{2}{c}{ Indeterminados } & \multicolumn{2}{c}{ Total } \\
& $\mathrm{n}$ & $\%$ & $\mathrm{n}$ & $\%$ & $\mathrm{n}$ & $\%$ & $\mathrm{n}$ & $\%$ \\
\hline Infante & 0 & 0 & 0 & 0 & 1 & 3,4 & 1 & 3,4 \\
Juvenil & 0 & 0 & 0 & 0 & 3 & 10,3 & 3 & 10,3 \\
Adulto joven & 2 & 6,9 & 0 & 0 & 3 & 10,3 & 5 & 17,2 \\
Adulto & 8 & 27,6 & 4 & 13,8 & 8 & 27,6 & 20 & 69,0 \\
Total & 10 & 34,5 & 4 & 13,8 & 15 & 51,7 & 29 & 100,0 \\
\hline
\end{tabular}

\section{Lesiones óseas y dentales identificadas}

La reacción periostal fue la lesión ósea más frecuente. Se analizaron los huesos largos de 15 individuos y nueve $(60,0 \%)$ presentaron esta formación de hueso nuevo, pero en estado inactivo (Fig. 2a). La exostosis auditiva fue la segunda lesión ósea más frecuente (30,7\%). Al evaluar los canales auditivos de 13 individuos, en cuatro se identificó este crecimiento óseo benigno (Fig. 2b). En dos de ellos, un femenino y un masculino, la exostosis auditiva se observó en ambos canales auditivos, mientras que en los otros dos individuos solo afectó un oído.

De los 29 individuos estudiados, 13 conservaron el cráneo, pero solo dos estaban

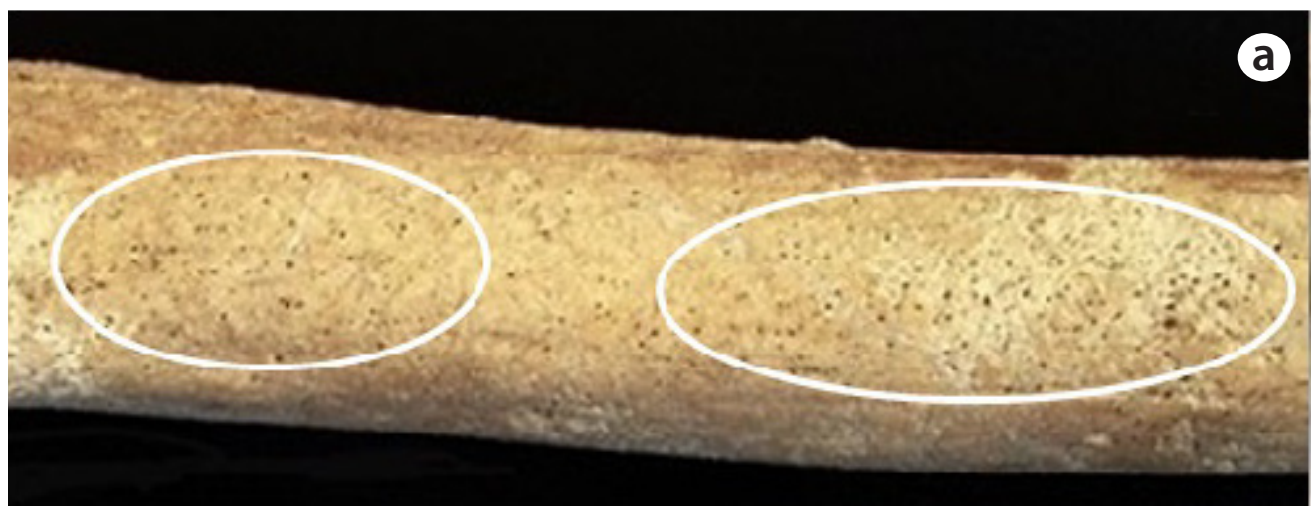

FIGURA 2a. Reacción periostal inactiva en fémur derecho (adulto femenino). 


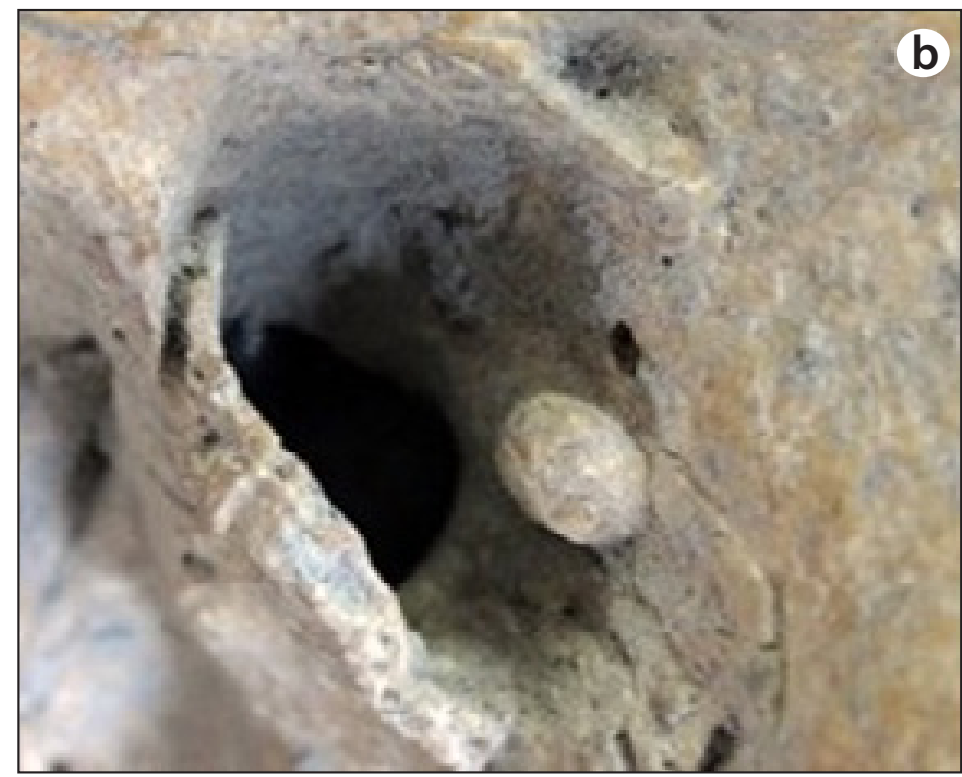

FIGURA 2b. Exostosis auditiva en canal auditivo izquierdo (adulto masculino).

completos. Los demás se encontraban incompletos y fragmentados. En dos $(15,4 \%)$ individuos se observó criba orbitaria: en uno de ellos la lesión era bilateral y estaba activa (Fig.3a) y en el otro solo se presentó en la órbita izquierda y la lesión estaba sana (Fig. 3b). Además, es importante mencionar que en ningún individuo se observó hiperostosis porótica y esto se puede deber al limitado estado de conservación de los restos óseos, el cual no permitió evaluar esta lesión en todos los individuos (Salazar, 2016).

Con respecto a las lesiones osteoarticulares, se analizaron las articulaciones entre los cuerpos vertebrales de 10 individuos. En dos (20,0\%) de ellos se registraron osteofitos en los cuerpos de las vértebras cervicales, torácicas y lumbares.

En la serie esqueletal estudiada, veinticinco individuos $(n=25)$ conservaron piezas dentales en las que se observaron caries, cálculos e hipoplasia del esmalte. Las caries y los cálculos fueron las patologías dentales más comunes. El 64,0\% de los individuos presentó caries (Fig.4a) y el $52,0 \%$, cálculos (Fig.4b). Además, se analizaron los incisivos y caninos superiores e inferiores (permanentes) de diecinueve individuos y siete (36,8\%) de ellos presentaron hipoplasia del esmalte.

Se analizaron los alvéolos de diecisiete individuos $(n=17)$. En doce $(n=12 ; 70,6 \%)$ de ellos se observó la pérdida de piezas dentales ante mortem y nueve $(n=9 ; 52,9 \%)$ tenían lesiones periapicales. Adicionalmente, en nueve individuos $(n=9)$ se evaluaron los márgenes bucales de los alvéolos y todos presentaron enfermedad periodontal generalizada.

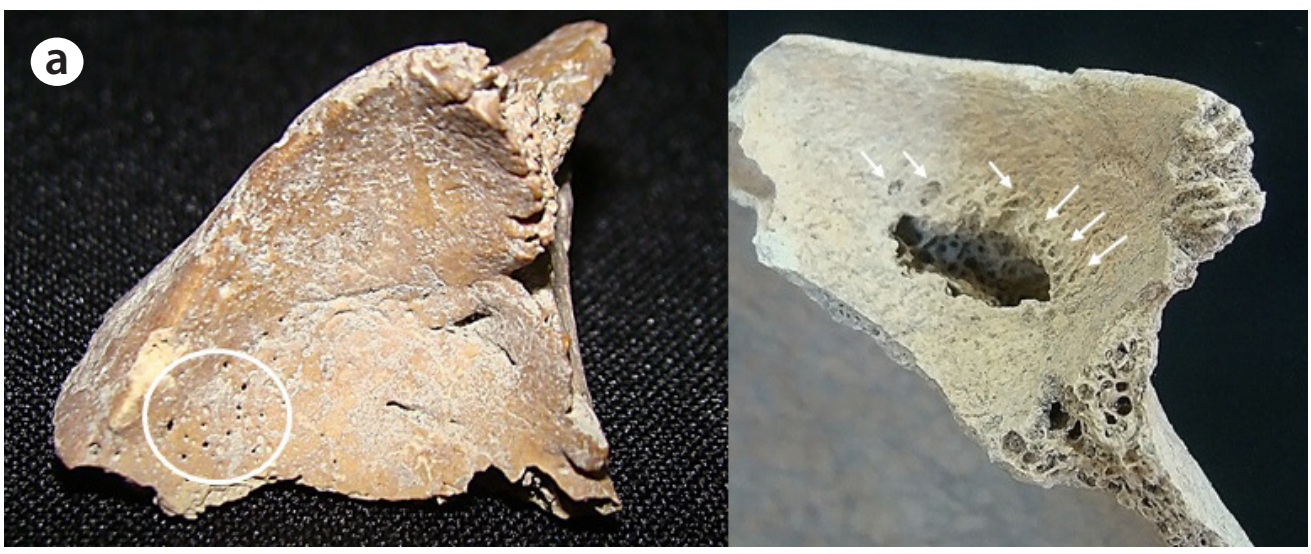

FIGURA 3a. Criba orbitaria activa, orbita izquierda (infante). FIGURA 3B. Criba orbitaria inactiva, orbita izquierda (adulto joven femenino). 


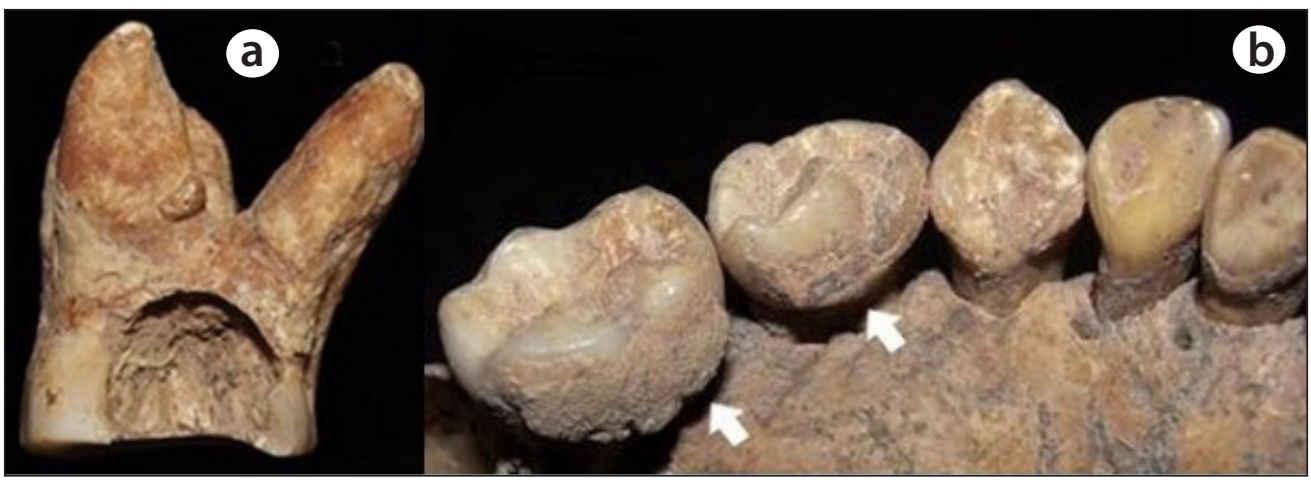

FIGURA 4a. Caries en primer molar superior izquierdo (adulto femenino). FIGURA 4b. Cálculos en molares inferiores izquierdos (juvenil)

\section{Frecuencia de las lesiones óseas y dentales según el sexo y la categoría de edad}

Al comparar la frecuencia de todas las lesiones óseas y dentales entre los individuos femeninos y masculinos, la prueba exacta de Fisher demostró que no hay una diferencia estadísticamente significativa $(p=0,69)$. En la Tabla 2 se presentan la frecuencia de cada lesión según el sexo de los individuos.

Con respecto a la frecuencia de todas las lesiones óseas y dentales entre las cuatro categorías de edad, sólo se observó una diferencia significativa entre los individuos adultos y los infantes $(p=0,02)$. Entre las demás categorías de edad no se registró una diferencia significativa (Tabla 3). En la Tabla 4 se detalla la frecuencia de las lesiones en cada categoría.

Tabla 2. Frecuencia de las lesiones óseas y dentales según el sexo de los individuos

\begin{tabular}{ccccccccc}
\hline \multirow{2}{*}{ Lesión } & \multicolumn{2}{c}{ Femenino } & \multicolumn{2}{c}{ Masculino } & \multicolumn{2}{c}{ Indeterminado } & \multicolumn{2}{c}{ Total } \\
& $\mathrm{n} / \mathrm{N}$ & $\%$ & $\mathrm{n} / \mathrm{N}$ & $\%$ & $\mathrm{n} / \mathrm{N}$ & $\%$ & $\mathrm{n} / \mathrm{N}$ & $\%$ \\
\hline Caries & $9 / 10$ & 90,0 & $3 / 4$ & 75,0 & $4 / 15$ & 26,7 & $16 / 25$ & 64,0 \\
$\begin{array}{c}\text { Pérdida de dientes ante } \\
\text { mortem }\end{array}$ & $8 / 10$ & 80,0 & $3 / 4$ & 75,0 & $1 / 15$ & 6,7 & $12 / 17$ & 70,6 \\
Periodontitis & $5 / 10$ & 50,0 & $4 / 4$ & 100,0 & 0 & 0 & $9 / 9$ & 100,0 \\
Lesión periapical & $5 / 10$ & 50,0 & $3 / 4$ & 75,0 & $1 / 15$ & 6,7 & $9 / 17$ & 52,9 \\
Reacción periosteal & $5 / 10$ & 50,0 & $2 / 4$ & 50,0 & $2 / 15$ & 13,3 & $9 / 15$ & 60,0 \\
Cálculos dentales & $5 / 10$ & 50,0 & $1 / 4$ & 25,0 & $7 / 15$ & 46,7 & $13 / 25$ & 52,0 \\
Hipoplasia del esmalte & $2 / 10$ & 20,0 & $2 / 4$ & 50,0 & $3 / 15$ & 20,0 & $7 / 19$ & 36,8 \\
Exostosis auditiva & $1 / 10$ & 10,0 & $3 / 4$ & 75,0 & 0 & 0 & $4 / 13$ & 30,7 \\
Lesión osteoarticular & $2 / 10$ & 20,0 & 0 & 0 & 0 & 0 & $2 / 10$ & 20,0 \\
Criba orbitaria & $1 / 10$ & 10,0 & 0 & 0 & $1 / 15$ & 6,7 & $2 / 13$ & 15,4 \\
\hline
\end{tabular}

Tabla 3. Comparación de valores de p del total de lesiones óseas y dentales entre las cuatro categorías de edad

\begin{tabular}{ccccc}
\hline & Infante & Juvenil & Adulto joven & Adulto \\
\hline Infante & $/$ & & & \\
Juvenil & $\mathrm{p}=0,71$ & $/$ & & \\
Adulto joven & $\mathrm{p}=0,57$ & $\mathrm{p}=0,98$ & $/$ & $/$ \\
Adulto & $\mathrm{p}=0,02$ & $\mathrm{p}=0,66$ & $\mathrm{p}=0,21$ & \\
\hline
\end{tabular}


Tabla 4. Frecuencia de las lesiones óseas y dentales según la categoría de edad

\begin{tabular}{|c|c|c|c|c|c|c|c|c|c|c|}
\hline \multirow{2}{*}{$\begin{array}{l}\text { Lesión/ } \\
\text { anormalidad }\end{array}$} & \multicolumn{2}{|c|}{ Infante } & \multicolumn{2}{|c|}{ Juvenil } & \multicolumn{2}{|c|}{ Adulto joven } & \multicolumn{2}{|c|}{ Adulto } & \multicolumn{2}{|c|}{ Total } \\
\hline & $\mathrm{n} / \mathrm{N}$ & $\%$ & $\mathrm{n} / \mathrm{N}$ & $\%$ & $n / N$ & $\%$ & $n / N$ & $\%$ & $\mathrm{n} / \mathrm{N}$ & $\%$ \\
\hline Caries & 0 & 0 & $1 / 3$ & 33,3 & $2 / 5$ & 40,0 & $13 / 20$ & 65,0 & $16 / 25$ & 64,0 \\
\hline Cálculos dentales & 0 & 0 & $2 / 3$ & 66,7 & $3 / 5$ & 60,0 & $8 / 20$ & 40,0 & $13 / 25$ & 52,0 \\
\hline $\begin{array}{l}\text { Pérdida de dientes } \\
\text { ante mortem }\end{array}$ & 0 & 0 & 0 & 0 & $1 / 5$ & 20,0 & $11 / 20$ & 55,0 & $12 / 17$ & 70,6 \\
\hline Lesión periapical & 0 & 0 & $1 / 3$ & 33,3 & 0 & 0 & $8 / 20$ & 40,0 & $9 / 17$ & 52,9 \\
\hline Periodontitis & 0 & 0 & 0 & 0 & $1 / 5$ & 20,0 & $8 / 20$ & 40,0 & $9 / 9$ & 100,0 \\
\hline $\begin{array}{l}\text { Hipoplasia del } \\
\text { esmalte }\end{array}$ & 0 & 0 & $1 / 3$ & 33,3 & $2 / 5$ & 40,0 & $4 / 20$ & 20,0 & $7 / 19$ & 36,8 \\
\hline Reacción periosteal & 0 & 0 & $1 / 3$ & 33,3 & $3 / 5$ & 60,0 & $5 / 20$ & 25,0 & $9 / 15$ & 60,0 \\
\hline Criba orbitaria & $1 / 1$ & 100,0 & 0 & 0 & $1 / 5$ & 20,0 & 0 & 0 & $2 / 13$ & 15,4 \\
\hline Exostosis auditiva & 0 & 0 & 0 & 0 & 0 & 0 & $4 / 20$ & 20,0 & $4 / 13$ & 30,7 \\
\hline $\begin{array}{l}\text { Lesión osteoar- } \\
\text { ticular }\end{array}$ & 0 & 0 & 0 & 0 & 0 & 0 & $2 / 20$ & 10,0 & $2 / 10$ & 20,0 \\
\hline
\end{tabular}

\section{DISCUSIÓN}

\section{Sobre las lesiones óseas y dentales identificadas}

Entre los individuos analizados, la caries fue la patología dental más frecuente. La presencia de esta lesión se relaciona con el consumo de carbohidratos, los cuales forman parte esencial de una dieta agrícola (Glassman y Garber, 1999; Hillson, 2008). El maíz y sus derivados, el frijol y la calabaza son alimentos ricos en carbohidratos originarios del Occidente de Mesoamérica (Zizumbo-Villareal y Colunga-García Marín, 2010; Zizumbo-Villarreal et al., 2012). En el sitio Los Limones, la ingesta de este tipo de alimentos se podría evidenciar con la presencia de metates, manos de moler y molcajetes, ya que estos artefactos son utilizados en el procesamiento y la maceración de alimentos como el maíz (Salazar, 2016).

En dos sitios arqueológicos de la cuenca de México: Cuicuilco (650-300 a.C.) (Márquez, 2006) y San Gregorio Atlapulco (1350-1521 d.C.) (Hernández, 2006), cuya principal estrategia de subsistencia fue la agricultura, también se reportó una alta frecuencia de caries. No obstante, es importante tomar en cuenta el consumo de otros alimentos cariogénicos, además de aquellos derivados de la agricultura. Por ejemplo, la población del sitio Xcambó en la costa Maya era sedentaria y dependía principalmente del maíz, pero, el incremento de caries durante el periodo Clásico tardío (550-750 d.C.) se atribuye al acceso a nuevos recursos alimenticios (locales o importados) y al consumo de bebidas alcohólicas cariogénicas (Cucina et al., 2011). De igual manera, en el sitio Terminal de Gas Natural Licuado, ubicado en la costa de Manzanillo, Colima, el aumento de caries durante las fases Colima-Armería (500-1100 d.C.) se vinculó con la introducción de nuevos alimentos (Hernández, 2012). Además, en el Occidente de Mesoamérica se ha reportado la elaboración de bebidas fermentadas a base de maíz y agave (Colunga-García Marín y Zizumbo-Villarreal, 2007; Zizumbo-Villarreal et al., 2012), las cuales se podrían relacionar con la formación de caries en los antiguos habitantes del sitio Los Limones.

Los individuos estudiados presentaron otras patologías dentales que pudieron ser causadas por caries en sus estadios más severos, como es el caso de las lesiones periapicales (Ortner, 2003) y la pérdida de piezas dentales ante mortem (Vega y Cucina, 2011). No obstante, es importante aclarar que las caries no es la única causa de estas lesiones, ya que 
también influyen otros factores como, por ejemplo, la falta de higiene bucal, la periodontitis y el desgaste dental (Brothwell, 1993; Roberts y Manchester, 2010; Waldron, 2009).

La periodontitis puede ser ocasionada por una higiene bucal deficiente, el desgaste dental y la irritación producida por los cálculos dentales (Brothwell, 1993; Waldron, 2009). Estos depósitos de placa calcificada son atribuidos a una dieta rica en carbohidratos y/o proteínas (Roberts y Manchester, 2010). De tal manera, la presencia de estas dos lesiones podría sugerir que los habitantes del sitio Los Limones consumían alimentos ricos en carbohidratos y en proteínas.

La hipoplasia del esmalte dental es causada por factores sistémicos como las deficiencias nutricionales (Pindborg, 1982; Seow, 2014; Wong, 2014). La dieta agrícola se ha asociado con este deterioro en la calidad nutricional, dado que se caracteriza por depender de un número limitado de plantas domesticadas (Larsen, 1995). Sin embargo, esta tendencia no es universal y se han observado variaciones. Por ejemplo, en los grupos con agricultura no-intensiva (ca. 1400 a.C.) e intensiva (ca. 500 a.C.) del valle de Oaxaca se reportó una alta frecuencia de hipoplasia del esmalte, a pesar de que la dieta fue variada y la intensificación de la agricultura no provocó un deterioro significativo en la salud de estas poblaciones (Hodges, 1987). De tal manera, es pertinente tomar en cuenta que los defectos en el desarrollo de los dientes son generalmente inespecíficos y pueden estar relacionados con una gran variedad de perturbaciones sistémicas (Kreshover, 1960), como las anomalías cromosómicas, las enfermedades infecciosas, las alteraciones neurológicas y los problemas metabólicos congénitos (Pindborg, 1982; Seow, 2014; Wong, 2014). Entre los individuos analizados, un adulto presentó hipoplasia del esmalte y, además, criba orbitaria en estado inactivo. Probablemente, tanto este individuo como los demás que tuvieron defectos en el esmalte dental sufrieron alguna perturbación sistémica de etiología inespecífica.

La criba orbitaria se identificó en dos individuos y puede estar relacionadas con diferentes insuficiencias nutricionales (Brickley, 2018) que causan anemia, escorbuto, raquitismo y osteomalacia (Brickley, 2018; Mays, 2018; Klaus, 2017; Oxenham y Cavill, 2010; Rivera y Mirazón, 2017; Snoody et al., 2018). Estas lesiones también pueden ser ocasionadas por condiciones neoplásicas, enfermedades infecciosas, lesiones traumáticas (Ortner, 2003), infecciones respiratorias (O'Donnell et al., 2020) y malaria (Gowland y Western, 2012).

Las condiciones de vida insalubres también causan deficiencias nutricionales, ya que pueden generar infecciones gastrointestinales que facilitan la pérdida de nutrientes, principalmente durante el destete (Olivares et al., 2002; Walker et al., 2009). Este pudo ser el caso del infante de $9 \pm 3$ meses que se analizó en esta investigación, el cual posiblemente estaba en el proceso de complementar la lactancia materna con otros alimentos. Este subadulto tenía criba orbitaria en estado activo en ambas órbitas esto indica que la enfermedad estaba presente al momento de la muerte. Por el contrario, la criba orbitaria que se identificó en el adulto joven femenino se encontraba inactiva y demuestra que la enfermedad había sido superada antes de morir. Sin embargo, no fue posible determinar la etiología específica de estas lesiones.

La reacción periostal fue la lesión ósea más frecuente entre los individuos analizados $y$, generalmente, ha sido atribuida a enfermedades infecciosas, entre otros factores (Roberts 2019; Weston 2012). El incremento de la población y el estilo de vida sedentario provocaron hacinamiento y problemas sanitarios. Estas condiciones pudieron facilitar la propagación de patógenos capaces de producir diferentes enfermedades infecciosas y parasitarias (Barret y Armelagos, 2013; Larsen, 2006; Ledger et al., 2019). En dos poblaciones de la cuenca de México: Tlajinga 33 (250-600 d.C.) y Cholula (900-1551 d.C.), se 
reportó una alta frecuencia de esta lesión, la cual se vinculó con infecciones producidas por los problemas sanitarios que pudieron tener estos centros urbanos como, por ejemplo, una mayor producción de basura y el consumo de agua contaminada (Márquez et al., 2002). Dado que los antiguos habitantes del sitio Los Limones también tuvieron un estilo de vida sedentario, es probable que las infecciones inespecíficas fueron la principal causa de las reacciones periostales identificadas. Asimismo, es relevante tomar en cuenta que estas lesiones pueden ser provocadas por enfermedades congénitas, metabólicas, genéticas y traumatismos (Chen et al., 2012; DeWitte, 2014).

La exostosis auditiva se relaciona principalmente con actividades acuáticas que implican el contacto con agua a baja temperatura y viento frío (Okumura et al., 2007; Pezo et al., 2009; Smith-Guzmán y Cooke, 2019). Es probable que los individuos analizados hayan realizado este tipo de actividades, ya que en los alrededores del sitio Los Limones se localizan ríos y lagunas, en los cuales se pudo haber practicado la pesca y la recolección de moluscos. Además, la presencia de exostosis auditiva en ambos sexos demuestra que las actividades acuáticas no fueron exclusivas de un solo género (Salazar, 2016). En el sitio Tlatilco también se reportó exostosis auditiva en mujeres y hombres (Márquez, 2006).

La relación entre la exostosis auditiva y la realización de actividades acuáticas sugiere el consumo de alimentos ricos en proteínas. Aunado a lo anterior, la alta frecuencia de cálculos dentales entre los individuos analizados hace referencia al acceso de este tipo de alimentos (ricos en proteínas). En el sitio El Tropel (ca. 300-700 d.C.), también ubicado en el valle de Colima, se efectuaron análisis de isótopos estables de Carbono y Nitrógeno que demostraron la ingesta de carne, pescado y/o moluscos, así como de maíz (Jácome y Morland, 2011). Por lo tanto, la identificación de estas lesiones podría indicar que los antiguos habitantes del sitio Los Limones no dependieron exclusivamente de la agricultura.

Por último, los osteofitos en los cuerpos vertebrales fueron la lesión menos frecuente en la serie esquelética estudiada y se relacionan con la degeneración de los discos intervertebrales, la cual es provocada por el envejecimiento, la carga mecánica excesiva, la herencia genética, la actividad física y la nutrición inadecuada (Adams y Roughley, 2006; Jurmain, 1999). En el sitio Los Limones, se observó esta lesión en dos mujeres mayores de 30 años y es posible que la edad haya sido el principal factor que causó su formación.

\section{Sobre la distribución de las lesiones óseas y dentales según el sexo, la edad y el lugar de inhumación}

No se observó una diferencia significativa entre individuos masculinos y femeninos al comparar la frecuencia de todas las lesiones óseas y dentales. Por lo tanto, la frecuencia de las lesiones identificadas no está determinada por el sexo de los individuos.

Con respecto a las categorías de edad, sólo se encontró una diferencia significativa al comparar todas las lesiones óseas y dentales entre los adultos y los infantes, quienes representan los dos extremos de los grupos etarios. Esto podría hacer referencia a la fragilidad o susceptibilidad que tiene cada individuo para enfermarse y morir (Wood et al., 1992), ya que el infante (9 meses \pm 3 meses) tenía criba orbitaria en estado activo al momento de morir. Por lo tanto, se podría mencionar que este individuo tuvo una mayor fragilidad en comparación con aquellos adultos que tuvieron lesiones en estado inactivo, las cuales reflejan que estos individuos superaron el o los eventos de "estrés" y sobrevivieron hasta alcanzar una edad más avanzada.

Por otra parte, al comparar las lesiones identificadas en los individuos inhumados 


\section{AGRADECIMIENTOS}

Las autoras les agradecen a las doctoras Yahaira Núñez y Carmen Grace Salazar por la revisión y las valiosas aportaciones que efectuaron a este documento. También extienden un agradecimiento a las maestras Aurora Pérez y Feliana Muñoz y al maestro Said Hernández por las sugerencias efectuadas. Asimismo, les agradecen a los revisores anónimos por las observaciones y recomendaciones que realizaron, ya que éstas permitieron mejorar el documento. en la unidad habitacional con los de las zonas funerarias, se observó que las patologías dentales como las caries, las lesiones periapicales y la pérdida de piezas dentales ante mortem, afectaron a todos los individuos, independientemente del lugar de inhumación. Esto podría indicar que los antiguos habitantes del sitio Los Limones tuvieron un acceso equitativo a los recursos alimenticios, en comparación con sitios del área Maya donde se ha reportado una diferencia relevante entre el sexo, el estatus social, la obtención de los recursos y la frecuencia de las patologías dentales (Cucina y Tiesler, 2003).

\section{CONCLUSIONES}

La integración de tres niveles de análisis: las lesiones óseas y dentales identificadas, las características ambientales del valle de Colima y los datos disponibles del contexto arqueológico, permitió sugerir que los habitantes del sitio Los Limones no basaron su subsistencia solamente en la agricultura. Tomando en cuenta la riqueza natural y la diversidad de ecosistemas terrestres y marinos que tiene el valle de Colima, es probable que sus antiguos pobladores hayan consumido una gran variedad de alimentos. De esta manera, estos individuos pudieron haber tenido una estrategia de subsistencia mixta, la cual incorporó la agricultura, la pesca, la caza y la recolección de plantas y frutos silvestres.

Algunas de las lesiones óseas y dentales identificadas, como las caries, los cálculos dentales, las recciones periostales y la exostosis auditiva, podrían reflejar alteraciones en la salud que se derivaron de esta estrategia de subsistencia. No obstante, es necesario llevar a cabo estudios complementarios, como la toma de radiografías, análisis de isótopos estables, estudios histológicos y análisis de ADN de patógenos, para establecer con mayor certeza la etiología de estas lesiones. Adicionalmente, es fundamental efectuar investigaciones paleobotánicas, paleoambientales y zooarqueológicas para conseguir una comprensión más amplia y detallada de las consecuencias que pudo provocar esta estrategia de subsistencia mixta en la salud de los antiguos habitantes del sitio Los Limones.

\section{LITERATURA CITADA}

Adams, M. A. y Roughley, P. (2006). What is intervertebral disc degeneration, and what causes it? Spine, 31(18), 2151-2161. https://doi.org/10.1097/01.brs.0000231761.73859.2c

Almendros, L. y González, F. (2009). El Occidente de México. La reocupación del valle de Colima. Boletín Americanista, 59, 137-154. Recuperado en https://revistes.ub.edu/index.php/BoletinAmericanista/article/view/13192/16531

Barret, R. y Armelagos, G. J. (2013). An unnatural history of emerging infections. Oxford, Inglaterra: Oxford University Press.

Beekman, C. S. (2010). Recent research in Western Mexican Archaeology. Journal of Archaeological Research 18, 41-109. https://doi.org/10.1007/s10814-009-9034-x

Brickley, M. (2018). Criba orbitalia and porotic hyperostosis: A biological approach to diagnosis. American Journal of Physical Anthropology, 167 (4), 896-902. https://doi.org/10.1002/ajpa.23701

Brooks, S. y Suchey, J. M. (1990). Skeletal age determination based on the os pubis: a comparison of the Acsádi-Nmeskéri and Suchey-Brooks methods. Human Evolution 5, 227-238. https://doi. org/10.1007/BF02437238

Brothwell, D. (1993). Desenterrando huesos: la excavación, tratamiento y estudio de restos del esqueleto humano. Madrid, España: Fondo de Cultura Económica.

Buckberry, J. y Chamberlain, A. T. (2002). Age estimation from the auricular surface of the ilium: a revi- 
sed method. American Journal of Physical Anthropology, 119 (3), 231-239. https://doi.org/10.1002/ ajpa. 10130

Buikstra, J.E. y Ubelaker, D. H. (1994). Standards for Data Collection from Human Skeletal Remains: Proceedings of a Seminar at the Field Museum of Natural History. Indianapolis, Estados Unidos: Western Newspaper Company.

Chen, E. M., Masih, S., Chow, K., Marcuk, G. y Patel, D. (2012). Periosteal reaction: review of various patterns associated with specific pathology. Contemporary Diagnostic Radiology, 35 (17), 1-5. https:// doi.org/10.1097/01.CDR.0000418465.87546.2d

Cohen, M. N. y Armelagos, G. J. (1984). Paleopathology at the origins of agriculture. New York, Estados Unidos: Academic Press.

Cohen, M. N. y Crane-Kramer, G. M. M. (2007). Ancient health: skeletal indicators of agricultural and economic intensification. Gainesville, Estados Unidos: University Press of Florida.

Colunga-García Marín, P. y Zizumbo-Villareal, D. (2007) Tequila and other Agave spirits from West-central Mexico: current germplasm diversity, conservation and origin. Biodiversity and Conservation, 16, 1653-1667. https://doi.org/10.1007/s10531-006-9031-z

Cucina, A., Perera, C., Sierra, T. y Tiesler, V. (2011). Carious lesions and maize consumption among the prehispanic Maya: an analysis of a coastal community in Northern Yucatan. American Journal of Physical Anthropology 145(4), 560-567. https://doi.org/10.1002/ajpa.21534

Cucina, A. y Tiesler, V. (2003). Dental caries and antemortem tooth loss in the Northern Peten area, Mexico: a biocultural perspective on social status differences among the Classic Maya. American Journal of Physical Anthropology, 122 (1), 1-10. https://doi.org/10.1002/ajpa.10267

DeWitte, S. N. (2014). Differential survival among individuals with active and healed periosteal new bone formation. International Journal of Paleopathology, 7, 38-44. https://doi.org/10.1016/j. ijpp.2014.06.001

Glassman, D. y Garber, J. (1999). Land use, diet, and their effects on the biology of the prehistoric Maya of Northern Ambergris Cay, Belize. En C. D. White (Ed.), Reconstructing ancient Maya diet (pp.119132). Salt Lake City, Estados Unidos: The University of Utah Press.

Goodman, A. H. y Martin, D. L. (2002). Reconstructing health profiles from skeletal remains. En R. H. Steckel y J. C. Rose (Eds.), The backbone of history. Health and nutrition of the Western hemisphere (pp.11-60). New York, Estados Unidos: Cambridge University Press. https://doi.org/10.1017/ CBO9780511549953.003

Gowland, R. L. y Western, A. G. (2012). Morbidity in the Marshes: using spatial epidemiology to investigate skeletal evidence for malaria in Anglo-Saxon England (AD 410-1050). American Journal of Physical Anthropology, 147(2), 301-311. https://doi.org/10.1002/ajpa.21648

Hernández, J. J. (2012). Prácticas funerarias entre los antiguos habitantes de la zona costera de Manzanillo, Colima. El caso del sitio arqueológico Terminal de Gas Natural Licuado (Tesis de licenciatura). Licenciatura de Antropología Física, Escuela Nacional de Antropología e Historia, Ciudad de México.

Hernández, P. O. (2006). Entre flores y chinampas: la salud de los antiguos habitantes de Xochimilco. En L. Márquez y P. O. Hernández (Eds.), Salud y sociedad en el México prehispánico y colonial (pp.327-365). Ciudad de México, México: Instituto Nacional de Antropología e Historia.

Hillson, S. (2001). Recording dental caries in archaeological human remains. International Journal of Osteoarchaeology, 11, 249-289.doi: 10.1002/oa.538. https://doi.org/10.1002/oa.538

Hillson, S. (2008). Dental pathology. En M. A. Katzenberg y S. R. Saunders (Eds.), Biological anthropology of the human skeleton (pp.301-340). New Jersey, Estados Unidos: John Wiley \& Sons.

Hodges, D. C. (1987). Health and agricultural intensification in the prehistoric valley of Oaxaca, Mexico. America Journal of Physical Anthropology, 73 (3), 323-332.https://doi.org/10.1002/ajpa.1330730305

Instituto Nacional para el Federalismo y Desarrollo Municipal. (2016). Enciclopedia de los Municipios 
y Delegaciones de México. Estado de Colima. Recuperado de http://www.inafed.gob.mx/work/ enciclopedia/EMM06colima/municipios/06010a.html

Jácome, C. A. y Morland, F. (2011) Maíz, agaves, venados y perros. Dieta, adaptación y sociedad de los antiguos habitantes del occidente de México. Estudio bioarqueológico de un sitio del Clásico mesoamericano, Villa de Álvarez, Colima. VI Foro Colima y su región. Arqueológica, antropología e historia, Colima. Recuperado de https://www.culturacolima.gob.mx/imagenes/foroscolima/6/5.pdf

Jurmain, R. (1999). Stories from the skeleton. Behavioral reconstruction in human osteology. New York, Estados Unidos: Taylor \& Francis Group.

Kelly, I. (1980). Ceramic sequence in Colima: Capacha, an early phase. Anthropological Papers of The University of Arizona 37. Arizona, Estados Unidos: The University of Arizona Press. https://doi. org/10.1086/jar.37.1.3629519

Klales, A. R., Ousley, S. D. y Vollner, J. M. (2012). A revised method of sexing the human innominate using Phenice's nonmetric traits and statistical methods. American Journal of Physical Anthropology, 149(1), 104-114. https://doi.org/10.1002/ajpa.22102

Klaus, H. D. (2017). Paleopathological rigor and differential diagnosis: case studies involving terminology, description, and diagnostic frameworks for scurvy in skeletal remains. International Journal of Paleopathology 19, 96-110. https://doi.org/10.1016/j.ijpp.2015.10.002

Kreshover, S. (1960). Metabolic disturbances in tooth formation. Annals New York Academy of Sciences, 85, 161-67. https://doi.org/10.1111/j.1749-6632.1960.tb49954.x

Larsen, C. S. (1995). Biological changes in human populations with agriculture. Annual Review of Anthropology, 24, 185-213. https://doi.org/10.1146/annurev.an.24.100195.001153

Larsen, C. S. (2006). The agricultural revolution as environmental catastrophe: Implications for health and lifestyle in the Holocene. Quaternary International, 150 (1), 12-20. https://doi.org/10.1016/j. quaint.2006.01.004

Ledger, M. L., Anastasiou, E., Shillito, L. M., Mackay, H., Bull, I. D., Haddow, S. D., ... Mitchell, P. D. (2019). Parasite infection at the early farming community of Çatalhöyük. Antiquity, 93(369), 573-587. https://doi.org/10.15184/aqy.2019.61

López, R. (2011). Los Limones II: Un espacio funerario en la región de Colima (Tesis de licenciatura). Facultad de Antropología, Universidad Veracruzana, Xalapa.

Lovejoy, O. C. (1985). Dental wear in the Libben population: its functional pattern and role in the determination of adult skeletal age at death. American Journal of Physical Anthropology 68,47-56. https://doi.org/10.1002/ajpa.1330680105

Manchester, K., Ogden, A. y Storm, R. (2016). Nomenclature in Palaeopathology. Paleopathology Newsletter 175, 1-51. Recuperado de https://paleopathology-association.wildapricot.org/resources/ Documents/Nomenclature\%20in\%20Palaeopathology\%20Web\%20Document.pdf

Márquez, L. (2006). La transición de la salud en Tlatilco y el surgimiento del estado en Cuicuilco. En L. Márquez y P. O. Hernández (Eds.), Salud y sociedad en el México prehispánico y colonial (pp.151210). Ciudad de México, México: Instituto Nacional de Antropología e Historia.

Márquez, L., McCaa, R., Storey, R. y del Ángel, A. (2002). Health and nutrition in pre-hispanic Mesoamerica. En R. H. Steckel y J. C. Rose (Eds.), The backbone of history. Health and nutrition of the Western hemisphere (pp. 307-338). New York, Estados Unidos: Cambridge University Press. https:// doi.org/10.1017/CBO9780511549953.014

Mays, S. (2018). Micronutrient deficiency diseases: anemia, scurvy, and rickets. En W. Trevathan (Ed.), The international encyclopedia of biological anthropology (pp. 1-5). https://doi. org/10.1002/9781118584538.ieba0271

O'Donnell, L., Hill, E.C., Anderson, A.S. y Edgar, H.J.H. (2020). Cribra orbitalia and porotic hyperostosis are associated with respiratory infections in a contemporary mortality sample from New Mexico. American Journal of Physical Anthropology. https://doi.org/10.1002/ajpa.24131 
Ogden, A. (2008). Advances in the Paleopathology of Teeth and Jaws. En R. Pinhasi y S. Mays (Eds.), Advances in Human Paleopathology (pp. 283-307). Chichester: Inglaterra: John Wiley and Sons. https://doi.org/10.1002/9780470724187.ch13

Okumura, M. M. M., Boyadjian, C. H. C. y Eggers, S. (2007). Auditory exostoses as an aquatic activity marker: a comparison of coastal and inland skeletal remains from tropical and subtropical regions of Brazil. American Journal of Physical Anthropology, 132(4), 558-567. https://doi.org/10.1002/ajpa.20544

Olay, M. A. y López, R. (2008). El valle de Colima y sus aldeas, una visión arqueológica de su historia antigua a través de varios rescates y salvamentos. Tomo VIII. Informe técnico final de los trabajos de evaluación arqueológica realizados durante la primera y segunda etapas del fraccionamiento Los Limones, Municipio de Villa de Álvarez, Colima (9 de Julio al 5 de octubre 2007 y 23 de octubre al 14 de diciembre de 2007). Colima, Centro INAH.

Olivares, J. L., Fernández, R., Fleta, J., Ruiz M. Y. y Clavel, A. (2002). Vitamin B12 and folic acid in children with intestinal parasitic infection. Journal of the American College of Nutrition, 21(2), 109-113. https://doi.org/10.1080/07315724.2002.10719202

Ortner, D. J. (2003). Identification of pathological conditions in human skeletal remains. San Diego, Estados Unidos: Academic Press. https://doi.org/10.1016/B978-012528628-2/50060-0, https://doi. org/10.1016/B978-012528628-2/50043-0

Oxenham, M. F. y Cavill, I. (2010). Porotic hyperostosis and criba orbitalia: the erythropoietic response to iron-deficiency anaemia. Anthropological Science, 118(3), 199-200. https://doi.org/10.1537/ ase.100302

Pezo, L., Pezo, S. y Eggers, S. (2009). Exostosis auditiva como marcador osteológico de actividad acuática en poblaciones formativas de la costa norte del Perú. Paleopatología 6,1-18. Recuperado de https://webs.ucm.es/info/aep/paleopatologia/vol6/exostosis.pdf

Pindborg, J. J. (1982). Aetiology of developmental enamel defects not related to fluorosis. International Dental Journal, 32(2), 123-134.

Pinhasi, R., y Stock, J.T. (Eds.). (2011). Human bioarchaeology of the transition to agriculture. Chichester, Inglaterra: John Wiley \& Sons. https://doi.org/10.1002/9780470670170

Piperno, D. R., Ranere, A. J., Holst, I., Iriarte, J. y Dickau, R. (2009). Starch grain and phytolith evidence forearly ninth millennium B.P. maize from the Central Balsas river valley, Mexico. Proceedings of the National Academy of Sciences 106(13), 5019-5024. https://doi.org/10.1073/pnas.0812525106

R Core Team. (2019). R: a language and environment for statistical computing. R Foundation for Statistical Computing. https://www.R-project.org/

Ranere, A. J., Piperno, D. R., Holst, Dickau, R. I., y Iriarte, J. (2009). The cultural and chronological context of early Holocene maize and squash domestication in the Central Balsas river valley, Mexico. Proceedings of the National Academy of Sciences 106(13), 5014-5018. https://doi.org/10.1073/ pnas.0812590106

Rivera, F., y Mirazón, M. (2017). New evidence suggesting a dissociated etiology for cribra orbitalia and porotic hyperostosis. American Journal of Physical Anthropology, 164(1), 76-96. https://doi. org/10.1002/ajpa.23258

Roberts, C. (2015). What did agriculture do for us? The bioarchaeology of health and diet. En G. Baker y C. Goucher (Eds.), The Cambridge world history. A world with agriculture 12.000 BCE-500 CE (pp.93-123). Cambridge, Inglaterra: Cambridge University Press. https://doi.org/10.1017/ CBO9780511978807.005

Roberts, C. (2019). Infectious disease: introduction, periostosis, periostitis, osteomyelitis, and septic arthritis. En J. E. Buikstra (Ed.), Ortner's identification of pathological conditions in human skeletal remains (pp.285-319). San Diego, Estados Unidos: Academic Press. https://doi.org/10.1093/infdis/ jiz148

Roberts, C. y Connell, B. (2004). Guidance on recording paleopathology. En M. Brickley y J. I. McKinley (Eds.), Guidelines to the Standards for Recording Human Remains (pp.34-39). Southampton, Ingla- 
terra: British Association for Biological Anthropology and Osteoarchaeology and Institute of Field Archaeologists. IFA papers (7).

Roberts, C. y Manchester, K. (2010). The archaeology of disease. Gloucestershire, Inglaterra:The History Press.

Salazar, M. M. (2016). Un acercamiento a las condiciones de salud y nutrición de un segmento poblacional del sitio arqueológico Los Limones, Colima (Tesis de maestría). Posgrado de Antropología Física, Escuela Nacional de Antropología e Historia, Ciudad de México.

Schaefer, M., Black, S. y Scheuer, L. (2009). Juvenile osteology. A laboratory and field manual. San Diego: Estados Unidos, Academic Press. https://doi.org/10.1016/B978-0-12-374635-1.00002-3

Scientific Working Group for Forensic Anthropology. (2010). Sex assessment. Recuperado de https:// www.nist.gov/sites/default/files/documents/2018/03/13/swganth_sex_assessment.pdf

Seow, W. K. (2014). Developmental defects of enamel and dentine: challenges for basic science research and clinical management. Australian Dental Journal, 59(1), 143-154. doi: 10.1111/adj.12104. https://doi.org/10.1111/adj.12104

Smith-Guzmán, N. E., y Cooke, R. G. (2019). Cold-water diving in the tropics? External auditory exostoses among the pre-Columbian inhabitants of Panama. American Journal of Physical Anthropology, 168(3), 448-458. https://doi.org/10.1002/ajpa.23757

Snoody, A. M. E., Buckley, H. R., Elliot G.E., Standen, V.G., Arriaza, B.T. y Halcrow, S.E. (2018). Macroscopic features of scurvy in human skeletal remains: A literature synthesis and diagnostic guide. American Journal of Physical Anthropology, 167(4), 876-895. https://doi.org/10.1002/ajpa.23699

Steckel, R. H., Larsen, C.S., Sciulli, P. W. y Walker, P. L. (2011). Data Collection Codebook. The Global History of Health Project, Ohio. Recuperado de https://www.uv.es/paleolab/Codebook-08-25051\%5B1\%5D.pdf

Steckel, R. H., Sciulli, P. W. y Rose, J. C. (2002). A health index from skeletal remains. En R.H. Steckel y J.C. Rose (Eds.), The backbone of history. Health and nutrition of the Western hemisphere (pp. 61-93). New York, Estados Unidos: Cambridge University Press. https://doi.org/10.1017/ CBO9780511549953.004

Ubelaker, D. H. (1978). Human skeletal remains. Excavation, analysis, interpretation. Chicago, Estados Unidos: Aldine Publishing Company.

Vega, E. y Cucina, A. (2011). Las patologías orales: caries, abscesos y pérdida de dientes en vida. En A. Cucina (Ed.), Manual de antropología dental (pp.107-129). Mérida, México: Universidad Autónoma de Yucatán.

Waldron, T. (2009). Paleopathology. Cambridge: Inglaterra, Cambridge University Press.

Walker, P. L. (2005) Greater sciatic notch morphology: sex, age, and population differences. American Journal of Physical Anthropology, 127(4), 385-391. https://doi.org/10.1002/ajpa.10422

Walker, P. L. (2008). Sexing skulls using discriminant function analysis of visually assessed traits. American Journal of Physical Anthropology, 136(1), 39-50. https://doi.org/10.1002/ajpa.20776

Walker, P. L., Bathurst, R. R., Richman, R., Gjerdrum, T. y Andrushko, V. A. (2009). The causes of porotic hyperostosis and cribra ortbitalia: A reappraisal of the iron-deficiency-anemia hypothesis. American Journal of Physical Anthropology, 139(2),109-125. doi: 10.1002/ajpa.21031. https://doi. org/10.1002/ajpa.21031

Weston, D. A. (2012). Nonspecific infection in paleopathology: interpreting periosteal reactions. En A. L. Grauer (Ed.), A companion to paleopathology (pp.492-512). Chichester, Inglaterra: Wiley-Brackwell. https://doi.org/10.1002/9781444345940.ch27

Wong, H. M. (2014). Aetiological factors for developmental defects of enamel. Austin Journal Anatomy, 1(1), 1003. https://hub.hku.hk/bitstream/10722/200426/1/Content.pdf?accept=1

Wood, J. W., Milner, G. R., Harpending, H. C., Weiss, K. M., Cohen, M. N., Eisenberg, L. E., ... Wilkinson, R. G. (1992). The osteological paradox: Problems of inferring prehistoric Health from skeletal samples. Currente Anthropology 33(4), 343-370. https://doi.org/10.1086/204084 
Revista Argentina de Antropología Biológica
Zizumbo-Villareal, D. y Colunga-García Marín, P. (2010). Origin of agriculture and plant domestication in West Mesoamerica. Genetic Resources and Crop Evolution 57, 813-825. https://doi.org/10.1007/ s10722-009-9521-4

Zizumbo-Villarreal, D., Flores-Silva, A. y Colunga-García Marín, P. (2012). The archaic diet in Mesoamerica: incentive for milpa development and species domestication. Economy Botany 66(4), 328-343. https://doi.org/10.1007/s12231-012-9212-5

Zuckerman, M. y Armelagos, G. J. (2011) The origins of biocultural dimensions in Bioarchaeology. En S. C. Agarwal y B. A. Glencross (Eds), Social Bioarchaeology (pp.15-43). Chichester, Inglaterra: Blackwell. https://doi.org/10.1002/9781444390537.ch2 